\title{
Fostering Engagement with Cultural Heritage through Immersive VR and Gamification
}

\author{
Stuart O’Connor, Simon Colreavy-Donnelly, and Ian Dunwell
}

\begin{abstract}
Digital games provide a recognised means of engagement and education when addressing challenges in educating and immersing individuals in their own heritages, and those of other cultures. Similarly, gamification techniques, commonly expressed as the addition of game elements to an existing process, have been successfully applied to augment existing resources and programmes. The many examples of gamification or serious games focusing on cultural heritage also highlight the potential benefits of using these principles for the purposes of supporting preservation and learning. In this chapter, we present I-Ulysses, a virtual-reality game designed to engage based around the notable work Ulysses by Irish author James Joyce. The rationale for the selection of Ulysses as a basis for the game's content and design was two-fold; firstly because of its cultural impact within Ireland, and secondly as its content appeared well-suited to exploration as a virtual reality experience. Facets of gamification are explored in I-Ulysses through key mechanics, including a focus towards virtual worlds and crowd intelligence based on real-world data, to highlight how these principles can be employed for cultural heritage preservation and knowledge transfer. Through feedback obtained from focus groups interacting with I-Ulysses, it can be seen that the gamified mechanics presented through the lens of virtual reality provide an informative and educational guide to Ulysses that would engage and appeal to a wide audience.
\end{abstract}

\footnotetext{
Dr. Stuart O'Connor

De Montfort University, Gateway House, Leicester, LE1 9BH, UK e-mail: stuart.oconnor@dmu.ac.uk

Dr. Simon Colreavy-Donnelly

De Montfort University, Gateway House, Leicester, LE1 9BH, UK e-mail: simon.colreavydonnelly@dmu.ac.uk

Dr. Ian Dunwell

Coventry University, Priory St, Coventry, CV1 5FB, UK e-mail: aa6537@ coventry.ac.uk
} 


\section{Introduction}

A holistic definition of cultural heritage includes both tangible and intangible societal attributes [2, 13]. Many digital tools focus towards the tangible, either virtually recreating historical sites, or providing remote access to archives and virtual museums [32, 33]. Games, similarly, have often focused on the educational aspects of tangible heritage, seeking to convey historical facts in an engaging fashion, whether through mobile apps, desktop games, and more recently virtual or augmented reality [17, 8, 31]. Games also exist addressing intangible attributes, which encompass practices, representations, expressions, knowledge, and skills passed down through generations. Projects such as e-Vita sought to allow older generations to communicate their lived experiences to youths through the form of digital games, taking these stories and adapting them to game-based narratives [24]. However, given the age-based aspects of the digital divide [25], it can easily be argued games are challenging, rather than solving, the issue of intergenerational transfer of cultural heritage. Nonetheless, storytelling is recognised as an established and vital means of conveying intangible heritage, either intergenerationally or cross-culturally [29]. Methods range from narratives of historical events, often told from a nuanced, individual perspective, though to fairy tales or fictions which often convey the moral principles of a culture. Whilst game-based learning need not require a narrative in all cases, its addition can, contextually, provide a powerful scaffold for engagement and immersion.

In the background of this chapter, existing work relating both serious and entertainment games to cultural heritage applications is explored. A particular emphasis is given to storytelling, and how games can provide an immersive backdrop against which a story can be told, and, perhaps more significantly, how they can provide a means of non-linear storytelling in which the player becomes an active participant in the narrative. Furthermore, by adopting procedural techniques for both foreground and background content, this non-linearity can become less of a choice of fixed paths, and rather an immersive and open-ended 'sandbox' experience. This leads to our cases in I-Ulysses, demonstrating the translation of a novel to a VR experience, and its facilitation through gamification.

\section{Background}

Gamification has been applied to cultural heritage in various forms throughout the years [5]. It is therefore important to consider the process of gamification and how it has been applied within the medium. This leads to identification in terms of design principles and the potential benefits for implementing such mechanics for cultural heritage. The applications of gamification however are varied, with some resultant games being purposed towards entertainment, while others focus on more serious pursuits, including preservation and education. By considering these all these 
elements, it serves to highlight how gamification can be a powerful tool towards engagement for specific purposes such as immersive VR experiences.

\subsection{Video Games}

Historical contexts and monuments have proved a rich source of inspiration for entertainment games, with examples such as Creative Assembly's Total War series, or Ubisoft's Assassin's Creed [3]. The latter gained recent media attention following the partial destruction of Notre Dame, as Ubisoft made one title which includes a detailed 3D replica of the cathedral free-to-play. This provides an interesting example of games providing a degree of digital preservation, and more generally how tangible heritage is being increasingly preserved in digital form outside of deliberate acts of preservation. Understandably, though, such games focus on entertaining the player, at the cost of historical accuracy, be this in terms of geography, language, culture, or mechanics. In such contexts, whilst they may facilitate and enable awareness, and engagement, detailed and accurate knowledge transfer is hard to assess or infer.

Entertainment games may have a closer connection to the intangible heritage present in many fairy tales, in that they often contain narratives with a strong moral undertone yet are set in a fictional environment. This can be as simple as 'good

vs evil', though, of course, nuances in culture can still be ascertained in terms of how protagonists and antagonists are portrayed. Game-based storytelling has also evolved: firstly towards non-linearity, and secondly towards moral ambiguity [9]. The former enables the player to shape their experience through the choices they make; the latter increases engagement with these choices by making them more complex than simply doing the 'good' or 'bad' thing, and instead encouraging them to apply their own judgement to moral dilemmas. This progression is partly a consequence of the maturation of games as a creative medium, but also reflects the increasing capacity of underlying technology to create richer, dynamic content.

Whilst objectives may differ, a wide range of entertainment game technologies have already brought benefits to cultural heritage. Not only do modern game engines such as Unity or Unreal expedite development; higher-level technologies often also have relevant applications. These include the use of tools for structuring narratives and objectives, which can often be straightforwardly repurposed to educational contexts, but also tools for procedural generation and the production of immersive environments. A key area of focus for this chapter is the use of crowd simulation to add plausibility and depth to environments, facilitating a transition from a static environment towards a more dynamic recreation of a setting. Implementing crowd simulations in a learning context requires consideration of the levels of interactivity required [23], which can range from a crowd service as a component of an immersive backdrop, though to explicit roles for crowds and characters as interactive agents.

The case study described in this chapter uses entertainment game technology to recreate a multi-linear narrative from James Joyce' Ulysses [15]. Before introducing this case alongside the role of crowd simulation in such a context, the next 
section considers, primarily from a pedagogical design standpoint, the specific use of 'serious' games when addressing the transfer of tangible and intangible heritage artefacts.

\subsection{Serious Games}

One could assume the role of 'serious' games when compared with entertainment games is to shift focus and emphasis towards accuracy and pedagogy; though a risk exists in compromising engagement, which is often the primary rationale for the use of game-based learning [20]. Existing examples of serious games in the area of cultural heritage often use fictional narratives, with tropes such as time-travel commonly applied as in the case of the Time Explorer game [27]. This is a common compromise due to the difficulty and expense involved with high-fidelity recreation, as well as the impossibility of creating a ubiquitously correct version of a historical setting. Whilst the games industry tends to delineate between pre-production and production in larger-scale projects, the smaller scale of most serious gaming ventures also means the cyclic approach effectively locks the game into pre-production as a prototype, rather than delivering a finished product. In a research sense, this affords opportunities to flexibly investigate and understand efficacy, however, engagement can also be difficult to assess using prototypical 'unpolished' products, given the frequent expectations of gamers towards high quality player experiences and fidelity.

Frameworks for the design, development, and evaluation of serious games in cultural heritage, such as that of Andreoli et al. [6], tend to emphasise interactive models that provide a cyclic basis for concept design, development, and evaluation. Whilst valid, these pose some inherent risks: in practical terms, finite resources and time-frames can limit iteration, and it is important to consider iteration as an approach with drawbacks as well as benefits, rather than purely as a conceptual ideal. Particular risks include the dilution of resources across multiple iterations, at the cost of fidelity in the end-product, and difficulty efficiently feeding back evaluation outcomes into pragmatic redesigns. This is particularly an issue for serious games as compared to entertainment games, as the simpler question of 'is it fun?', is typically compounded with transdisciplinary questions such as 'is it historically accurate?', 'is it pedagogically effective?', and 'does it engage the target audience?'.

A consideration with respect to fidelity is its complex relationship to learning outcomes. For simulator-based learning, a common assumption is that increased fidelity benefits learning outcomes. Fidelity itself is often separated into different subcategories [18], for example visual fidelity, in terms of how realistically a scene is rendered, through to interactive fidelity, in terms of how objects and processes operate and interact. This subcategorisation is useful when considering different facets of learning and engagement: a recreation of an ancient monument may benefit from high levels of visual fidelity, whereas a simulation of an interpersonal interaction or historical process can benefit more from interactive fidelity. Identifying where fidelity is non-critical can prove more valuable than identifying critical cases, not 
only because it allows resources to be focused appropriately, but also because it grants levity to the designer when seeking to provide an engaging experience.

Holistic consideration of fidelity beyond required learning outcomes is also worthy of consideration. When seeking to engage a target audience, consideration of their context of play is also essential. Target demographics that already engage widely with entertainment games can bring accompanying higher expectations of fidelity, and attempting to engage such groups in their leisure time is particularly risky in terms of successful reach. Often, the solution lies in the use of games as part of more structured or blended learning solutions, ameliorating the need to stimulate intrinsic motivation. However, as technology matures, it is increasingly feasible to provide high-fidelity recreations of sites and characters within the pragmatic constraints of a typical game-based learning project. In the next section, the i-Ulysses project is introduced, which sought to capitalise on these technologies to provide a gamified approach to learning cultural heritage within a virtual environment.

\section{3 i-Ulysses}

The I-Ulysses: Poetry in Motion project focused on development of a virtual-reality game, using the process of gamification to engage players with cultural heritage. James Joyce's Ulysses [14] is a culturally significant novel that takes place in the busy streets of Dublin circa 1904. The novel introduces spatial and auditory metaphors as core mechanics, with crowd psychology and behaviour utilised as narrative elements. I-Ulysses investigates the value of gamification and pedagogy to that end and for potential use in educating and preserving cultural heritage as an immersive experience. The project referenced the concepts of Derryberry, Salen and Zimmerman on meaningful play and serious learning in games; Dondlinger's work on virtual learning environments; Ellis' work on educational game design; and Norman's The Design of Everyday Things [11, 30, 35, 12, 19].

At its core, I-Ulysses adapts the Ulysses novel into a spatial virtual environment. Here the spatial and auditory aspects of the virtual environment are connected to specific audio-spatial events from the book, following the same method of employing sound cues as employed by Joyce in Ulysses. The main objectives of the project were:

- To educate players in the narrative and storytelling techniques of a classic literary work using an immersive virtual reality experience.

- To facilitate this learning, in an integrated, multi-modal manner that reflects key aspects of the specific novels structure.

- To enable players to consult contextual information about a book in a real-time context on a mobile platform, whilst traversing around the virtual environment it takes place in.

- To create an interplay between the themes of a book, the historical and physical environment it takes place in and the current virtual environment that the player inhabits. 
I-Ulysses was designed to be as close in relation to the novel as possible with its story presentation and core mechanics. In this regard, it follows a multi-linear story pattern through its virtual environment and the objectives contained therein. There are several bonus characters or stories that vary throughout the virtual environment depending on player choices (Bloom, Kernan, Blazes Boylan, The Secretary etc.). These characters stories can be accessed as bonus missions upon completing the final level, which takes place in the Martello Tower. The Martello Tower is like the central hub of the game; the means by which the other sections of the story are connected together and can be accessed from any direction in the level structure, so-to-speak. It should be noted that the story of the game is based on the 'Wandering Rocks' chapter of the book, but it interlinks events that take place throughout its story. Each back-story, or bonus level, comes with text taken from the relevant chapter. This presence may be an image, a sound or something that Joyce directly hints at in the text. These sections of the book take place on the busy streets of Dublin during the day and so the representation of crowd behaviour and psycho-dynamics is an aspect of the experience.

In addition to the directional prompts, the game incentivises the player to pursue specific goals, but does not constrain them to certain choices. The environment transitions from the city, to back stories in the Tower, Kildare, and The Chapel, and then reverts to the relevant 'Wandering Rocks' section of the city. The stream-ofconsciousness of each character in the book is represented by the players character being able to float around the scene in an unrestricted first-person perspective, rather than the more confined space of the city. When the player clicks on the text menu they are returned to the broader environment, which has been changed by their interaction in the back-story level, and is encouraged to look for other interaction events, which again reveal further dimensions of the story. In the flow chart below one set of connections and interaction outcomes, and the paths needed to unlock them, is represented (Figure 1).

Interactive elements within the environment help to provide a new means of understanding Ulysses. Within the environment certain events, such as back-stories and thought bubbles, appear at different times, depending on the choice of objectives that each character attempts to complete. This is illustrated with the time-keeping function of the clock, used to suggest that certain elements of the active environment can alter depending on player choices. In the city-version of the environment, the camera also changes its depth of field and moves closer or further away from the player depending on which character they are using, indicating that several different points of view on the environment are possible simultaneously. The thought bubble enables the player to see what the character is thinking based on the objectives and back stories which they have completed. An alternate viewport appears in the bottom right hand corner of the screen showing other characters paths. These features sought to more fully realise the interactive potential of the virtual reality and explore means by which interior monologue and multi-linear storytelling techniques of Ulysses could be transferred to an interactive experience.

The setting also sought to enforce the player's sense of objective, since certain choices are result in visible changes to the environment and affect what level they 


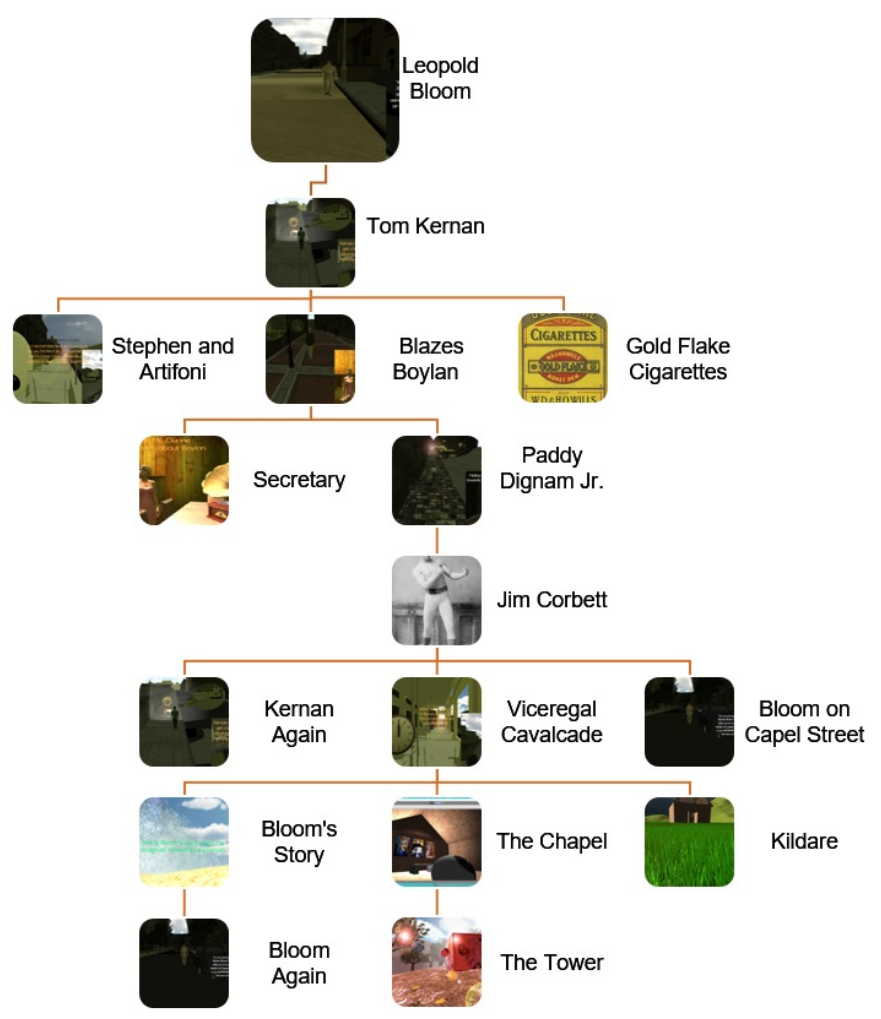

Fig. 1 Story nodes highlighting the path of interaction and connections between key events.

progress to, whilst others will not. This assumes the player will be more engaged with the environment, as they observe the relationship between their actions and consequences. The technique also sought to provoke interest in learning about the network that underpins the character's relationships. As shown above (Figure 1), completing interactions in a particular order will change the outward environment and the possibility for new interaction events to occur within specific missions. The environment emulates these effects in real-time, so that choices shape the player's understandings of time and the overall structure of the environment. In this way the player sees their own actions held in sharp relief against the other character's stories and the behaviour of the larger crowd on that day.

This real-time effect foregrounds the objectives of the environment; it focuses specifically on what drives or motivates the player in the environment and what mechanisms are being used to direct their attention. The real-time effect also focuses on how to make the player aware of the fact that they are being directed towards discrete learning goals, having both a specific and general goal. The objectivebased environment is themed around Joyce's use of key sound events and so the environment uses certain cues or tropes from Joyce's work, namely representing 
how sound cues interact with the interior monologue and multi-linear storytelling techniques. Use of songs mentioned in Joyce's work and advertising motifs help to establish the Victorian setting of the environment (such as the Gold Leaf Cigarettes advertisement for example), by establishing it within the time period using references from Ulysses.

As described in this section, I-Ulysses is a closely linked narrative embedded within a virtual environment. Establishing a sense of presence in this environment, whilst also creating an environment capable of supporting exploratory learning, required a degree of adaptivity and depth in the design. One particular need was to recreate an urban environment immersively; in particular, it was desirable to use crowds and crowd simulation. In the next section, we discuss an approach to crowd simulation in the Unity engine, as used for the I-Ulysses project, and describe its implementation to create plausible crowd behaviours based on human perception.

\section{Inhabited Virtual Dublin}

Producing realistic crowds to inhabit virtual locations consists of various challenges, due in part to the interlinked components that comprise a system for simulating emergent crowd behaviour [16, 34]. Contributing is the fact that a distinction can be made between crowds employed for serious applications [4] and those geared more towards entertainment purposes [10]. Crowds for serious applications are often modelled using crowd data [36] and have a limited amount of human interaction, being built towards specialised purposes. In contrast, virtual crowds utilised for entertainment are typically have a user-centered focus, wherein user interaction and perception are highly important considerations [22]. A clear example can be seen looking at crowds applied as interactive elements in video games, with developers stressing the importance of believability or perceived realism [7]. Serious games on the other hand, such as I-Ulysses, need to offer a middle ground between a serious focus and entertainment. I-Ulysses in particular, incorporates both elements from the real-world in the form of the novel and crowd data, as well as a design paradigm based in gamification and pedagogy to support engagement and learning.

For the development of the crowds, an adapted three-stage methodology was employed, covering analysis, synthesis, and perception. These stages, in more detail, are: (1) analysis of real-world instances of crowd behaviour; (2) synthesis of crowd behaviour into a simulation; and (3) perceptual evaluation of the resultant crowd behaviour. Related research has shown that conducting these three tasks is a necessity for producing a robust study that contains a model, which is 'grounded in reality' [26, 21]. By consulting crowd data to build the scenario and parameters it ensures this real-world grounding exists for the model and can be preserved.

For analysis, Temple Bar in the center of Dublin was selected due to its place within the Ulysses novel and the importance of dense crowds as a narrative mechanic. Temple Bar being pedestrianised and highly trafficked provides a source of high-density crowd data that can be applied to various locations within the virtual 
environment. In addition, by selecting typical pedestrianised streets, which are a prominent type of location utilised in both video games and serious applications, results can be applicable to a wide range of future endeavours. Other important factors for the selection of the location include; being a central location in a major city, with a viable perspective on the pedestrian lanes, having limited boundaries comparable to the size of the space, and being viable for reconstruction.

Crowd footage was acquired at peak times during clear weather from live web camera feeds that were a part of the EarthCam network [1]. A manual annotation was tailored towards the crowd configuration. In this process, an annotator manually adds semantically relevant descriptors. Generally, the annotation should be as simple and efficient as possible, only considering relevant factors [26]. This of particular importance when considering video footage, where different frames need to be analysed. Two variables of interest for I-Ulysses were group frequency, referring to the number of different groups that are present within the given scene, and group density, referring to the number of individuals in these groups. By annotating the groups using the mark-up method, values for these two variables are tagged and calculated at each key frame (Figure 2).

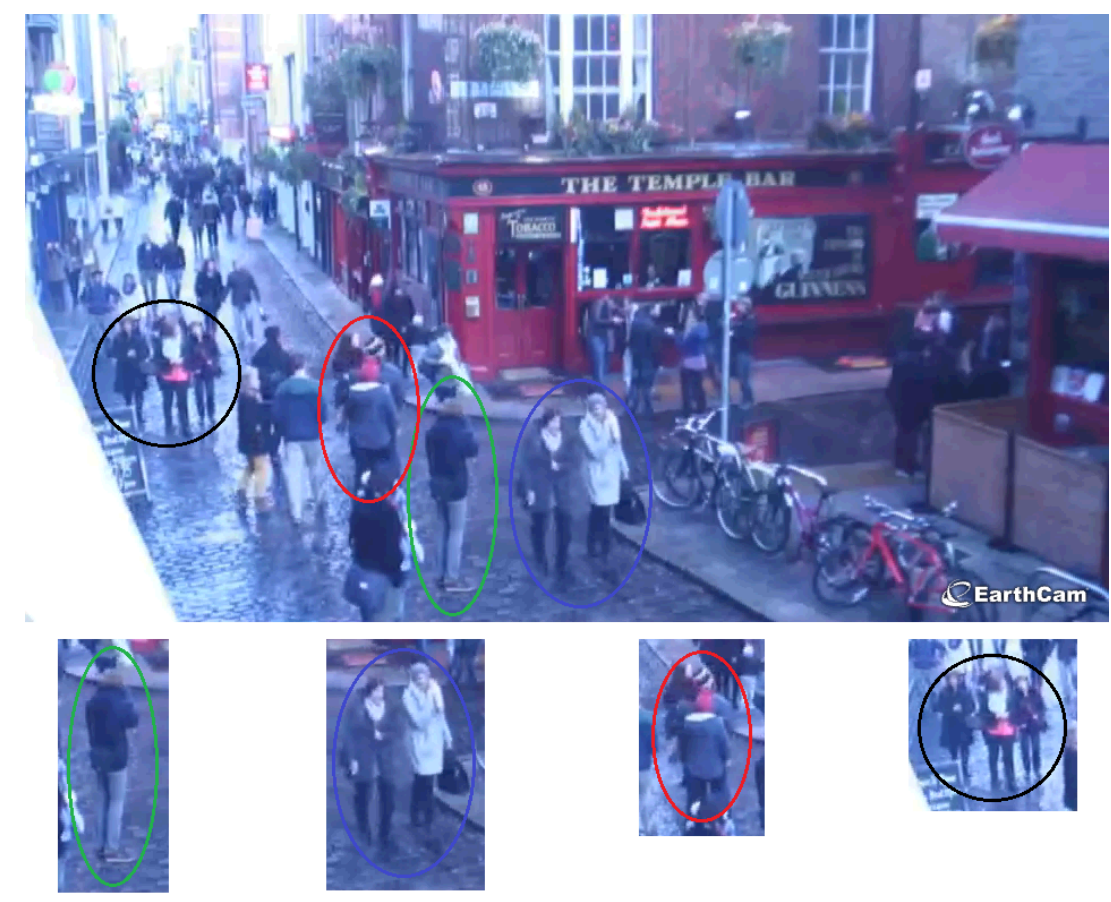

Fig. 2 A key frame from the Temple Bar crowd footage, showing an example of the annotation process whereby groups are tagged. The colour of the annotation denotes density of the group. 
The I-Ulysses virtual environment, objects, and mechanics were developed within the Unity game engine. Using the functionality of 'prefabs', a generic feature that allows a developer to group game objects together into packages that can be quickly instantiated in the game environment, development could be quickly accelerated as collections of assets could be packaged and modified for all instances simultaneously. This functionality enables a convenient hypostasis effect referred to as inheritance, where the designer can implement changes to a specific asset or can make changes backwards compatible to all instances of an asset.

For synthesis of crowds, an agent-based system was implemented to give their form of intelligence. Typically, an agent approach will include a form of decision making, pathfinding and steering, which allows agents to perceive, think, and act, to a limited extent. For the purposes of I-Ulysses, a focal point was given to group frequency and group density, so it was important to limit the behavioural variability of the agents for perceptual evaluation, as implementing more complex algorithms that consider more factors such as social forces, would potentially lead to emergent behaviour influencing results and detracting from the established narrative of Ulysses. As such, the sophistication of the agent system was kept suitably limited with the steering behaviours and overall group controller.

To limit emergent behaviour, a steering force based approached was utilised in a similar fashion Reynolds Boids [28]. This consisted of multiple steering behaviours to act as the main behavioural control system for the agents. Several key steering behaviours are initialised for each agent in a defined group:

- Cohesion: Determines how closely each member of the group sticks together.

- Alignment: Determines how closely the group follows in formation.

- Separation: Determines how far agents in the group can move apart from each other.

- Separation from other groups: Determines how the group as a whole move to avoid colliding with other groups.

- Path-following: Determines the predefined paths groups follow within the virtual environment to reach their intended destination. Through a corrective force pointing down the path, agents are able to respond dynamically and then navigate back onto course.

These rules consist of two key variables, a weight to determine how much impact a specific rule has in relation to the other rules, and a radius to determine the size of the local neighbourhood considered for that rule. The key differences to the Boids model are the additional rules and weighting factor, which prevents agents from exhibiting the flocking behaviour and instead become autonomous pedestrians in their select groups. To compliment this, a group controller allowed for the initialisation of the desired number of groups and their density (Figure 3), defined at runtime by an area calculated and visualised through a connected spline. Individual agents are selected from a large quantity of agent prefabs setup within a pool. In this manner, the majority of agents visible are unique so obviously repeated models are minimised within the scene. Each agent has a number of key variables, including a minimum and maximum speed, a specific size represented as a radius, a maximum force value 
and a mass. These variables are kept constant, as the focal point is towards group frequency and density for I-Ulysses and subsequent perceptual evaluation.

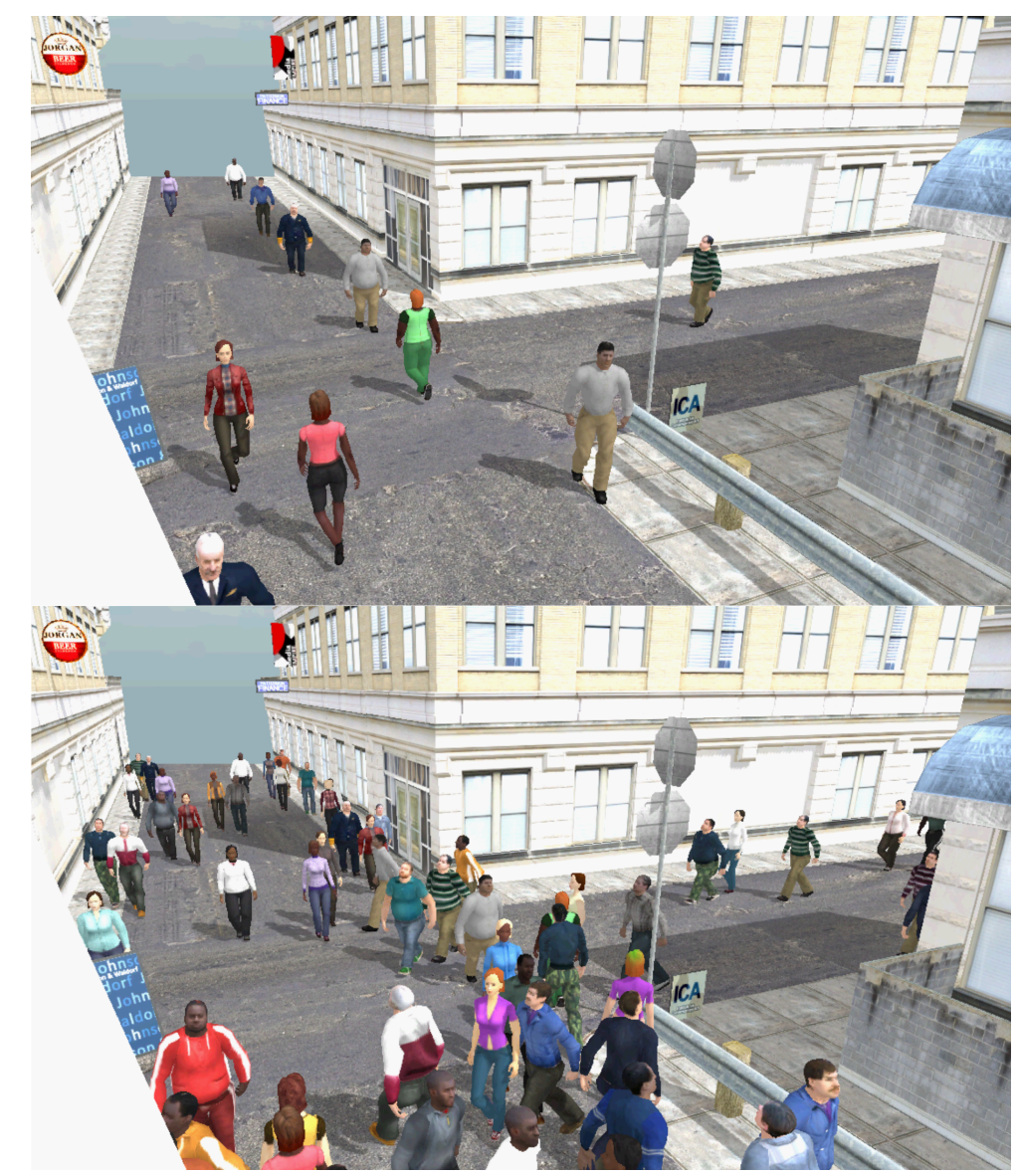

Fig. 3 Dublin, Temple Bar crowd simulation showing low density on the top and high density on the bottom.

For perception, psychophysical evaluation was conducted using stimuli recorded from runtime in a virtual recreation of the temple bar area as seen in the initial crowd footage (Figure 3). A total of seventy-eight participants ( 69 males; 9 females) (4 aged 13-18; 57 aged 19-25; 12 aged 26-35; 5 aged 36-50) registered responses through the online platform, for each of the experiment's twelve trials relating to the Dublin, Temple Bar location. The six group frequency trials were presented first, followed by the six group density trials. On each trial, participants were shown both the original and simulated video clips for a duration of 10-seconds. They were asked how realistic do you find the groups in the simulated video and then told to give a percentage before moving on the next trial. For each trial within a set, a different 
intensity was presented $(10 \%, 30 \%, 50 \%, 70 \%, 90 \%$ and 100\%), with 50\% intensity being the value extracted from the crowd data for that variable and the others being calculated from that. A lower intensity means less groups or fewer agents within each group, depending on the variable being examined, with a higher intensity signifying an opposite effect.

For group frequency in Temple Bar, the highest average response of 0.71 was for $70 \%$ intensity, equating to a value of 22 groups. For group density in Temple Bar, the highest average response of 0.88 was for $50 \%$ intensity, equating to a value of 2.3 agents per group. These results highlight what was perceived as being most realistic in terms of composition and are complimented through identification of absolute thresholds, which determine the point at which the composition stops being perceived as realistic. The absolute thresholds for group frequency in Temple Bar were identified as $35 \%$ intensity and $95 \%$ intensity, which links to a frequency of 12 and 31. In addition, the absolute thresholds for group density in Temple Bar were identified as $33 \%$ intensity and $81 \%$ intensity, which links to a density of 1.5 and 3.7 .

These results highlight some important considerations for I-Ulysses, in particular when examining a comparison between the crowd data and these perceptual results. It can be seen that perception of a realistic crowd is within a close range to the $50 \%$ extracted from the crowd data, with optimum density at 50\% and optimum frequency at a single increment of $70 \%$. Additionally, there is flexibility in terms of the thresholds, with the upper threshold for frequency as high as $95 \%$. This shows that participants were determining a larger number of agents within the scene to be within the realms of realism, where as reducing the number proved more problematic, with the lower threshold for density only as low as 33\%. By establishing these thresholds, the frequency and density of the crowds can be potentially increased to give a more immersive experience closer to Joyce's intentions with Ulysses, while keeping the crowds of realistic composition to ensure integrity is not comprised.

As previously noted for I-Ulysses, by employing serious elements with an emphasis towards accuracy and pedagogy, a risk exists in compromising engagement and it is therefore important to balance the mechanics for this type of application. In the next section, we discuss this balance through the lens of a story focused cross-section adapted for I-Ulysses and describe its reception for the purposes of engagement with cultural heritage.

\section{Story Cross-Section}

What follows is a specific example of a story cross-section, adapted from the book. In the following example, the player is introduced to some of the first instances of the interior monologue in the environment. The interior monologue is the mechanism by which Joyce shows us the inner-world and thoughts of the character. In the first segment we learn that Blazes Boylan is placing a bet on Sceptre, the Horse that was favourite to win the Ascot Cup in 1904. It is later revealed that Bloom 
had accidentally given a tip 'Throwaway' (when he told a friend to throwaway the newspaper, advertising the race), which was the name of the horse that actually went on to win the Cup, in 1904. Bloom walks past places in the environment that are mentioned in the dialogue (Figure 4). It is at this point that the player transitions between Boylan and Bloom, as playable characters.

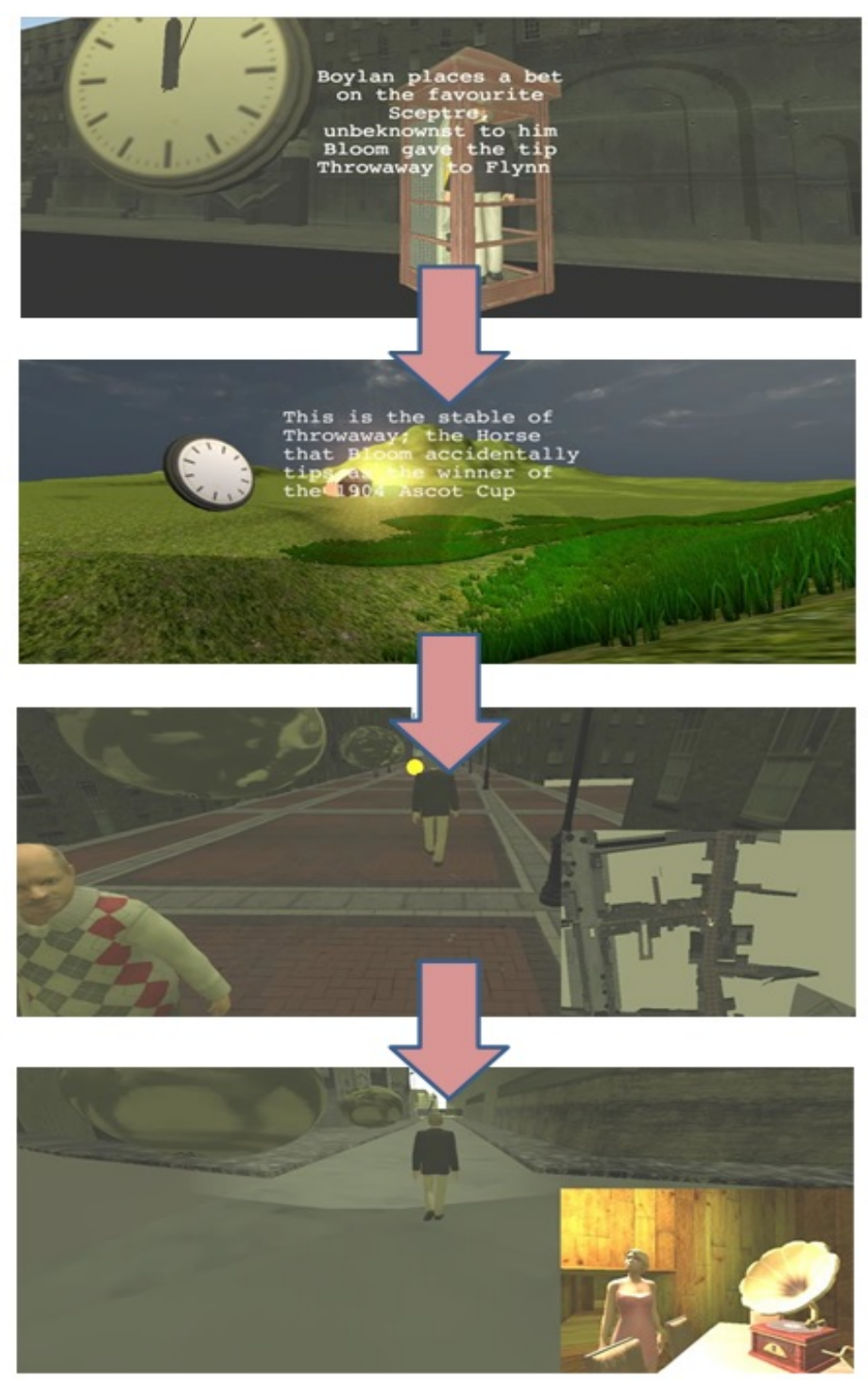

Fig. 4 An example story arc where Bloom walks past key locations in the environment that are discussed in the dialogue. 
This arc creates an important link between events from the book, because it is an example of Joyce's use of multiple points of view, or opinions, on the same events and characters. As the player progresses they gain insights from Boylan's secretary about his character, references to his gambling problems, with relevant dialogue and narration, cutting, like a film, to the description of the outside world, in the following sections. The concepts discussed in this cross-section form a part of the instructional mode of a 'blended learning' framework, wherein a tutor can read this section of the book, draw attention to the key techniques and then visually demonstrate this through having a student play the game. The benefit of having an interactive virtual model is that its use emphasizes the participatory learning dimensions of the blended learning framework. Additionally, I-Ulysses is designed to function independently as a virtual environment that a player can interact with and learn about Joyce's work from. This means that the operational aspects of the environment require a different set of developmental criteria from what would be involved in developing an educational website, an E-Book or a Digital Companion.

The I-Ulysses environment has a virtual design emphasis (Figure 5), unlike the Digital Companion and E-Book formats. By engaging with the virtual environment, the player learns about the book through the design elements of the environment itself. The virtual scenarios were based on the author's interaction with students and tutors, who have taught Joyce and were informed by several user-test samples and focus groups that concentrate on improving the interface by having it tested, as much as possible. In total there were seven samples; a total of eighteen students of English from National University of Ireland Galway, thirty students from the University of Vienna, nine students from the School of Computer Science, in Trinity College Dublin and members of the public on several occasions, at the James Joyce Centre in Dublin, Ireland. The first sample at the James Joyce Centre consisted of seven participants: they were Joyce enthusiasts, a journalist writing for a national newspaper and students at University College Dublin, studying Joyce. The subsequent samples consisted of seven and five participants respectively, all of whom were members of the general public with no extensive knowledge of Joyce. Each sample played the game and filled out a questionnaire, detailing areas that they felt needed improving.

It was noted that the main objective of the virtual environment would be to facilitate the player's understandings of the innovative storytelling techniques that Joyce employed in Ulysses. This list of learning outcomes was derived by compiling each of the unique storytelling devices employed by Joyce and dividing them into three broad effects or modes: the interior monologue (or thought bubble), the multi-linear storytelling technique and the use of back stories. The responses of the attendees from the focus groups created a re-evaluation of the initial stated learning outcomes, referred to now as learning objectives. By interacting with the environment, the players from the groups were better able to:

- Note the difference between interior monologue segments from Ulysses, the dialogue and the environment in a visualised, aural context.

- Discern the character's motives, in an educational context.

- Explore the back stories of each character in a way that connected their shared experiences to the wider environment of the city. 


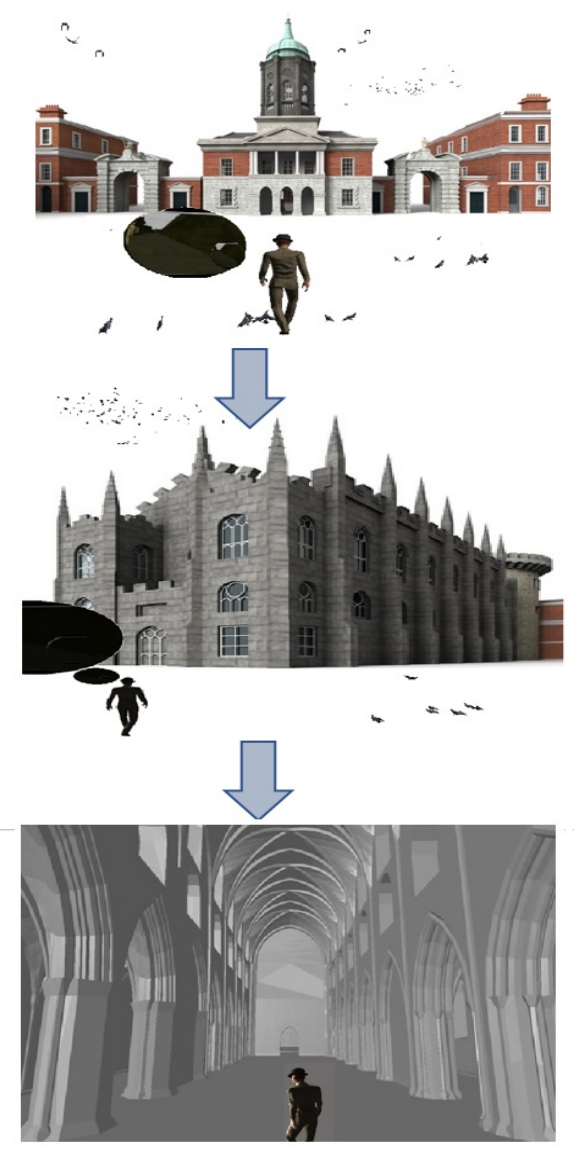

Fig. 5 Virtual environments showing Georgian-era Dublin Castle and Christ Church.

- Discover more about the cultural world of Joyce's Ulysses and Joyce's main works, in an educational context.

- Describe a multi-linear perspective on the events of the chapter, rather than focusing on a single story-facet at one time in one place.

- Describe the events of 'Wandering Rocks' in a way that emphasised the space, setting, use of sound effects and the interconnectedness of key events and characters from the book.

- Evaluate how Joyce used sound to connect events across different spatial, geographical and temporal locations in the city.

The respondent samples stated that being able to see the difference between the character's imagination and reality, while having corresponding thought bubbles to delineate these elements of the experience, made the experience more engaging and 
informative in the context of seeing the technique represented from the book in a virtual format.

The respondents also argued that they felt the use of the map and the presentation of the city provided a good overview of the space of the book and the character interactions occurring in it. The consensus was that the environment, from a graphical point of view, made an effective use of the urban environment and that it felt as if the player was walking around the actual space of Dublin. A respondent from the Vienna test noted that the ability to see the streets of Dublin in a visualised context, while also exploring the narrative of the book, made the experience of using the environment more engaging and was comparable to a walking guided tour of the area.

One of the primary areas of difficulty was assessing and operationalising the data extracted from the focus groups. In many cases the types of data gathered were not formatted for a statistical evaluation; rather than exploring the mean data, information and insight gathered was more anecdotal than procedural. As Sandoval, McKenny and Reeves argue, a virtual reality format lends itself to novel forms of education-design; but because the subject matter of the I-Ulysses project is esoteric, specifically in relation to Joyce's work, it is more difficult to assess the output in a scalable format like Conjecture Mapping.

Where the mapping format and education-design theories were useful, however, was in providing the template for an 'Iterative' design process, whereby the process and practice underwent significant revision, which integrated a discourse involving both the content of Joyce's work and the employment of virtual reality technologies. This is ultimately why I-Ulysses benefited from having explored commercial and technological underpinnings, in the EI-commercialisation study and academic placement, because they provided an opportunity to test the design-hypothesis and 'High-Level Conjecture' in a real-world setting.

Without this data and the data gained from the focus groups, the project could potentially have focused solely upon a single facet of the design-hypothesis at a given time. Where the I-Ulysses sought to break new ground was in offering the potential for the design-process to be externalised into the format of the learning environment; incorporating both the Joyce-focused and technology-focused aspects simultaneously. This externalisation provided another layer of engagement that reflects the type of continuous and recursive engagement that readers traditionally have with Ulysses.

The virtual reality setting is a means to draw a player into Joyce's work and involve them in the learning environment, with a distinct set of goals and criteria for progress. The main drawback of this approach, is attempting to find a 'best fit' for the game objectives of the environment and the literary nature of the work. In terms of the manner in which user-testing scenarios and focus groups were conducted, there was a fairly broach pitching of backgrounds and specialisms, however, ideally more user-testing scenarios and focus groups could have been conducted, to that end. Often times, the specialisms of literature and interactive digital media do not easily cross over and so it is not easy to find candidates intersecting with these areas. However, since the project was undertaken, general interest, awareness of 
digital technology, location-based augmented and virtual reality projects (Pokémon Go) has increased immensely among the younger generations, and so such a market would prospectively exist for the project now, in a way that was not the case when it was initially devised.

There are also potential areas for improving I-Ulysses; in terms of design emphasis and methodological focus, the primary objective of the project was to establish a functional interface for the implementation of 3D game assets that would resemble and reflect key elements of the content of Ulysses. However, there are other areas that could be focused on, in a more developed format. One such area is the use of sophisticated rendering technology to reproduce an architecturally authentic 'Georgian-era' Dublin, for the benefit of tourism and cultural heritage.

Another area that was explored was the granularity of the game-assets employed in its development; the manipulation of its pre-existing hierarchy, whereby the system could be updated in real-time and instantiate instances of player avatars or 'thought-bubbles', reflecting their own educational design-architecture and the student's engagement with the content. In the formats indicated below (Figure 6), it would be possible for the tutor to update modules for player avatar appearance; what the thought bubbles would show and to re-format aspects of the environments design and realisation, reflecting an ongoing discourse.

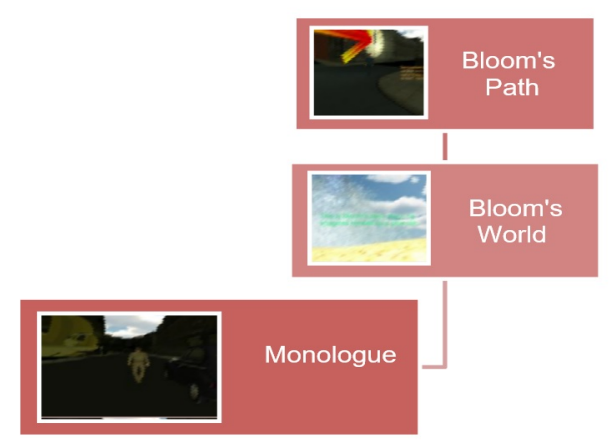

Fig. 6 Map of the key elements that the player can instantiate.

The data employed in this hierarchy would allow such to occur in an online serverbased context, thus expanding the horizons of I-Ulysses into something more closely resembling an online community, or an archive or repository of digital knowledge maintained by its subscribers. This educational-design architecture maps key features of the educational-design curriculums proposed by Sandoval, McKenny and Reeves; positioning them in a live, practical context, which could conceivably connect with other online resources. 


\section{Conclusions}

The main objective of I-Ulysses was to explore new ways for players to engage with the cultural heritage and learn about aspects of Joyce's work through an immersive VR experience. Key elements of Joyce's storytelling techniques were adapted and made conceptually easier for players to understand, balancing the accuracy and pedagogy of the serious elements with gamification. The virtual environment formed the crux of the various mechanics, with player agency at the forefront as they navigate through circa 1904 Dublin to interact with the works of Joyce and discover narrative connections. The virtual environment was supported through an exploration towards simulating realistic crowds within Dublin, to capture the tone from the novel whilst retaining perceptual plausibility. This served to highlight the importance of taking player perception into account and the utilisation of perceptual thresholds when developing a cross-purpose application, such as a serious game. Thus, the virtual environment is aimed to be educational in teaching Joyce and Ulysses, but it is also intended to be entertaining to support player immersion and engagement with cultural heritage materials.

Feedback from the user tests indicated that the I-Ulysses delivered in providing an informative, educational guide to Ulysses that would appeal to a range of players, from academics and casual readers of the book. The most important aspect to emphasise, however, is that while it represents a guide to the book, it is also primarily a virtual reality experience. The choice of a virtual reality model has the distinct advantages of separating physical space and narrative, enabling the player to interrogate the differences between historical, narrative space and physical space. One of the main objectives of the project was to provide the player with a virtual model that adapts the techniques that writers employ. The I-Ulysses environment remodels and remediates these techniques into a multi-linear, 3D game experience. The project researched the areas of overlap between games, virtual reality and literature. In this regard, it could form an effective template for adapting different kinds of literary narratives into virtual reality formats. The practical work done for I-Ulysses in Unity was about demonstrating how to do this most effectively, with an efficient use of resources to target a wide range of platforms, including those with limited specifications such as mobile devices.

\section{Acknowledgements}

Research on the I-Ulysses project was undertaken as part of the Digital Arts Humanities program between 2011 and 2015. An industrial/academic placement also took place in the third year, with the Computer Graphics Department (GV-2) at Trinity College Dublin, with Professor John Dingliana. During this placement, the author was given access to assets in Ogre from the Inside Joycean Dublin Project, a sister-project, and was tasked with gamifying them. The project was involved in 
a commercialisation spinout fast track (CFTD-1), with Enterprise Ireland's Kevin Burke, in the third year, and the involvement of the Inagh Valley Trust.

\section{References}

1. Earthcam network. Available online: http://www.earthcam.com/ (Accessed on 2017-04-25)

2. Unesco definition of cultural heritage. http://www.unesco.org/new/en/culture/themes/illicittrafficking-of-cultural-property/unesco-database-of-national-cultural-heritagelaws/frequently-asked-questions/definition-of-the-cultural-heritage/. Accessed: 2019-17-06

3. Assassins Creed: Unity. [PS3 DVD] (2014)

4. Almeida, J.E., Rosseti, R.J., Coelho, A.L.: Crowd simulation modeling applied to emergency and evacuation simulations using multi-agent systems. arXiv preprint arXiv:1303.4692 (2013)

5. Anderson, E.F., McLoughlin, L., Liarokapis, F., Peters, C., Petridis, P., De Freitas, S.: Developing serious games for cultural heritage: a state-of-the-art review. Virtual reality 14(4), 255-275 (2010)

6. Andreoli, R., Corolla, A., Faggiano, A., Malandrino, D., Pirozzi, D., Ranaldi, M., Santangelo, G., Scarano, V.: A framework to design, develop, and evaluate immersive and collaborative serious games in cultural heritage. J. Comput. Cult. Herit. 11(1), 4:1-4:22 (2017). DOI 10.1145/3064644. URL http://doi.acm.org/10.1145/3064644

7. Bernard, S., Therien, J., Malone, C., Beeson, S., Gubman, A., Pardo, R.: Taming the mob: Creating believable crowds in assassin's creed. In: Game Developers Conference (San Francisco, CA, Feb 18-22) (2008)

8. Boboc, R.G., Gîrbacia, F., Duguleană, M., Tavčar, A.: A handheld augmented reality to revive a demolished reformed church from braŞov. In: Proceedings of the Virtual Reality International Conference - Laval Virtual 2017, VRIC '17, pp. 18:1-18:4. ACM, New York, NY, USA (2017). DOI 10.1145/3110292.3110311. URL http://doi.acm.org/10.1145/3110292.3110311

9. Christiansen, P.: Designing ethical systems for videogames. In: Proceedings of the 12th International Conference on the Foundations of Digital Games, FDG '17, pp. 21:121:7. ACM, New York, NY, USA (2017). DOI 10.1145/3102071.3102088. URL http://doi.acm.org/10.1145/3102071.3102088

10. Cournoyer, F., Fortier, A.: Massive crowd on assassin's creed unity: Ai recycling. In: Game Developers Conference (San Francisco, CA, Mar 2-6) (2015)

11. Derryberry, A.: Serious games: Online games for learning (2004). URL http://www.adobe.com/resources/elearning/pdfs/serious-games-wp.pdf. Accessed: 2012-0725

12. Dondlinger, M.J.: Educational video game design: A review of the literature. Journal of applied educational technology 4(1), 21-31 (2007)

13. Doulamis, A.D., Voulodimos, A., Doulamis, N.D., Soile, S., Lampropoulos, A.: Transforming intangible folkloric performing arts into tangible choreographic digital objects: The terpsichore approach. In: VISIGRAPP (5: VISAPP), pp. 451-460 (2017)

14. Joyce, J.: Ulysses. London: Bodley Head (1969)

15. Joyce, J.: Ulysses. Simon \& Brown (2011)

16. Lerner, A., Chrysanthou, Y., Lischinski, D.: Crowds by example. In: Computer Graphics Forum, vol. 26, pp. 655-664. Wiley Online Library (2007)

17. Liarokapis, F., Petridis, P., Andrews, D., de Freitas, S.: Multimodal serious games technologies for cultural heritage. In: Mixed Reality and Gamification for Cultural Heritage, pp. 371-392. Springer (2017)

18. McMahan, R.P., Herrera, N.S.: Affect: Altered-fidelity framework for enhancing cognition and training. Frontiers in ICT 3, 29 (2016). DOI 10.3389/fict.2016.00029. URL https://www.frontiersin.org/article/10.3389/fict.2016.00029

19. Norman, D.: The design of everyday things: Revised and expanded edition. Basic books (2013) 
20. O’Connor, S., Doukianou, S., Awad, M., Dixon, R., O’Neill, D., Dunwell, I.: Developing gamified elements to influence positive behavioural change towards organisational energy efficiency. In: European Conference on Games Based Learning, pp. 488-497. Academic Conferences International Limited (2017)

21. O'Connor, S., Liarokapis, F., Jayne, C.: Perceived realism of crowd behaviour with social forces. In: Information Visualisation (iV), 2015 19th International Conference on, pp. 494499. IEEE (2015)

22. Pagulayan, R.J., Keeker, K., Wixon, D., Romero, R.L., Fuller, T.: User-centered design in games. CRC Press Boca Raton, FL (2002)

23. Panzoli, D., Peters, C., Dunwell, I., Sanchez, S., Petridis, P., Protopsaltis, A., Scesa, V., de Freitas, S.: Levels of interaction: A user-guided experience in large-scale virtual environments. In: Proceedings of the 2010 Second International Conference on Games and Virtual Worlds for Serious Applications, VS-GAMES '10, pp. 87-90. IEEE Computer Society, Washington, DC, USA (2010). DOI 10.1109/VS-GAMES.2010.27. URL https://doi.org/10.1109/VSGAMES.2010.27

24. Pappa, D., Dunwell, I., Protopsaltis, A., Pannese, L., Hetzner, S., de Freitas, S., RebolledoMendez, G.: Game-based learning for knowledge sharing and transfer: the e-VITA approach for intergenerational learning, pp. 974-1003. IGI Global (2011). DOI 10.4018/978-1-60960495-0.ch045. The full text of this item is not available from the repository.

25. Peters, A., Winschiers-Theophilus, H., Mennecke, B.: Bridging the digital divide through facebook friendships: A cross-cultural study. In: Proceedings of the 2013 Conference on Computer Supported Cooperative Work Companion, CSCW '13, pp. 249254. ACM, New York, NY, USA (2013). DOI 10.1145/2441955.2442014. URL http://doi.acm.org/10.1145/2441955.2442014

26. Peters, C., Ennis, C.: Modeling groups of plausible virtual pedestrians. IEEE Computer Graphics and Applications (4), 54-63 (2009)

27. Raptis, G.E., Fidas, C.A., Avouris, N.M.: Do field dependence-independence differences of game players affect performance and behaviour in cultural heritage games? In: Proceedings of the 2016 Annual Symposium on Computer-Human Interaction in Play, CHI PLAY '16, pp. 38-43. ACM, New York, NY, USA (2016). DOI 10.1145/2967934.2968107. URL http://doi.acm.org/10.1145/2967934.2968107

28. Reynolds, C.W.: Flocks, herds and schools: A distributed behavioral model. In: ACM Siggraph Computer Graphics, vol. 21, pp. 25-34. ACM (1987)

29. Rizvic, S.: Story guided virtual cultural heritage applications. Journal of Interactive Humanities 2 (2014)

30. Salen, K., Tekinbaş, K.S., Zimmerman, E.: Rules of play: Game design fundamentals. MIT press (2004)

31. Schofield, G., Beale, G., Beale, N., Fell, M., Hadley, D., Hook, J., Murphy, D., Richards, J., Thresh, L.: Viking vr: Designing a virtual reality experience for a museum. In: Proceedings of the 2018 Designing Interactive Systems Conference, DIS '18, pp. 805815. ACM, New York, NY, USA (2018). DOI 10.1145/3196709.3196714. URL http://doi.acm.org/10.1145/3196709.3196714

32. Styliani, S., Fotis, L., Kostas, K., Petros, P.: Virtual museums, a survey and some issues for consideration. Journal of cultural Heritage 10(4), 520-528 (2009)

33. Sylaiou, S., Mania, K., Paliokas, I., Pujol-Tost, L., Killintzis, V., Liarokapis, F.: Exploring the educational impact of diverse technologies in online virtual museums. International Journal of Arts and Technology 10(1), 58-84 (2017)

34. Thalmann, D., Musse, S.R.: Crowd Simulation, 2 edn., chap. State-of-the-Art, pp. 9-30. Springer (2012)

35. Willis, J.: Research-based strategies to ignite student learning: Insights from a neurologist and classroom teacher (2006)

36. Zhao, M., Cai, W., Turner, S.J.: Clust: Simulating realistic crowd behaviour by mining pattern from crowd videos. In: Computer Graphics Forum, vol. 37, pp. 184-201. Wiley Online Library (2018) 